\title{
Gene silencing of USP1 by lentivirus effectively inhibits proliferation and invasion of human osteosarcoma cells
}

\author{
JINBO LIU ${ }^{1}$, HONGJUN ZHU ${ }^{2}$, NING ZHONG ${ }^{3}$, ZIFENG JIANG ${ }^{4}$, LELE XU $^{5}$, \\ YOUPING DENG ${ }^{6}$, ZHENHUAN JIANG $^{7}$, HONGWEI WANG ${ }^{8}$ and JINZHI WANG ${ }^{9}$
}

\footnotetext{
${ }^{1}$ Department of Orthopaedics, The Third Affiliated Hospital of Soochow University, Changzhou, Jiangsu 213003;

${ }^{2}$ Department of Thoracic Surgery, The First People's Hospital of Shangqiu, Shangqiu, Henan 476100;

${ }^{3}$ Department of Thoracic Surgery, Kunshan First People's Hospital Affiliated to Jiangsu University, Kunshan,

Jiangsu 215000, P.R. China; ${ }^{4}$ Clinical Laboratories, The University of Chicago Medical Center, Chicago,

IL 60637, USA; ${ }^{5}$ Department of Gastroenterology, The First Affiliated Hospital of Soochow University,

Suzhou, Jiangsu 215200, P.R. China; ${ }^{6}$ Department of Medicine, Rush University Medical Center, Chicago,

IL 60612, USA; ${ }^{7}$ Department of Orthopaedics, People's Hospital of Yixing City, Yixing, Jiangsu 214200, P.R. China;

${ }^{8}$ Department of Medicine, University of Chicago, Chicago, IL 60637, USA; ${ }^{9}$ Department of Cell Biology,

School of Medicine, Soochow University, Suzhou, Jiangsu 215007, P.R. China
}

Received August 17, 2016; Accepted October 20, 2016

DOI: $10.3892 /$ ijo.2016.3752

\begin{abstract}
Osteosarcoma is the most frequent malignant bone tumor, affecting the extremities of adolescents and young adults. Ubiquitin-specific protease 1 (USP1) plays a critical role in many cellular processes including proteasome degradation, chromatin remodeling and cell cycle regulation. In the present study, we discovered that USP1 was overexpressed in 26 out of 30 osteosarcoma tissues compared to cartilage tumor tissues and normal bone tissues. We then constructed a lentiviral vector mediating RNA interference (RNAi) targeting USP1 and demonstrated that it significantly suppressed the mRNA and protein expression of the USP1 gene in U2OS cells. Knockdown of USP1 inhibited the growth and colonyforming, as well as significantly reduced the invasiveness of U2OS cells. Western blot analysis indicated that suppression of USP1 downregulated the expression of many proteins including SIK2, MMP-2, GSK-3 $\beta$, Bcl-2, Stat3, cyclin E1, Notch1, Wnt-1 and cyclin A1. Most of these proteins are associated with tumor genesis and development. RNAi of SIK2 significantly decreased SIK 2 protein expression and inhibited the ability of forming colonies, as well as induced apoptosis and reduced the invasiveness of U2OS cells. Collectively, our results suggest that silencing USP1 inhibits cell proliferation
\end{abstract}

Correspondence to: Dr Jinzhi Wang, Department of Cell Biology, School of Medicine, Soochow University, Suzhou, Jiangsu 215007, P.R. China

E-mail: 451588568@qq.com

Dr Hongwei Wang, Department of Medicine, University of Chicago, Chicago, IL 60637, USA

E-mail: hwang1@medicine.bsd.uchicago.edu

Key words: osteosarcoma, USP1, RNA interference, SIK2 and invasion in U2OS cells. Therefore, USP1 may provide a novel therapeutic target for the treatment of osteosarcoma.

\section{Introduction}

Osteosarcoma is the most common bone malignancy in the pediatric age group, with a very high propensity for local invasion and distant metastasis $(1,2)$. In recent years, despite the development of new multimodal therapeutics, the prognosis for patients with this disease is generally poor $(3,4)$. Therefore, effective therapeutic methods for osteosarcoma are urgently needed.

Ubiquitin (Ub), a kind of important signaling protein, is involved in many cellular processes, including cell cycle regulation, DNA damage response, chromatin remodeling and proteasome degradation. Similar to phosphorylation, ubiquitin modification is reversible, and deubiquitinases or DUBs are known to mediate ubiquitin removal (5). DUBs can remove $\mathrm{Ub}$ moieties from Ub-protein conjugates, resulting in reduced ubiquitination signaling (6).

The misexpression of DUBs has been demonstrated to be associated with a number of human diseases, especially cancers (7). Among them, USP1 is one of the best-characterized human DUBs, which is involved in the control of cell differentiation and plays an important role in the regulation of the cellular response to DNA damage (8).

USP1 has been reported to be overexpressed in several human malignant tumors $(9,10)$. Moreover, USP1 has been identified as the DUB responsible for deubiquitinating Fanconi anemia complementation group I (FANCI), Fanconi anemia group D2 (FANCD2) and proliferating cell nuclear antigen (PCNA) in the DNA damage response. PCNA is monoubiquitinated in response to DNA damage that stalls progression of the replication fork and then initiates recruitment of translesion DNA synthesis (TLS) DNA polymerases in the DNA 
damage tolerance pathway $(11,12)$. USP1 plays as a regulator of PCNA ubiquitination in the DNA damage response. It may deubiquitinate the DNA replication processivity factor, PCNA, thus, contributing to preventing unscheduled recruitment of error-prone TLS DNA polymerases, as a safeguard against error-prone TLS of DNA (13).

USP1-associated factor 1 (UAF1) stabilizes USP1 and allosterically increases its catalytic activity, which is very low in the absence of the cofactor $(14,15)$. In addition, UAF1 contributes to targeting USP1 to its nuclear substrates. USP1 performs its functions in the context of a heterodimeric complex with UAF1 (16).

RNAi is a powerful approach that silences the expression of endogenous genes via distinct messenger RNA degradation pathways. It may be a potential therapeutic agent for many kinds of diseases, including HIV, cerebrovascular and cardiovascular diseases, viral hepatitis, metabolic diseases, neurodegenerative disorders and cancers $(17,18)$.

In the present study, specific-shRNA with lentivirus vector was employed to knock down USP1 in osteosarcoma U2OS cells, and the effects of USP1 silencing on cell growth and invasion were explored.

\section{Materials and methods}

Main reagents. Mouse anti-human USP1 polyclonal antibody was purchased from the ProteinTech Group (Wuhan, China); mouse anti-human monoclonal antibody to $\mathrm{Bcl}-2$ and mouse anti-human polyclonal antibodies to MMP-2, GSK-3 $\beta$, Stat3, cyclin E1, Notch1, Wnt-1 and cyclin A1 were purchased from ImmunoWay Biotechnology Co. (Plano, TX, USA); MG132 and mouse anti-human monoclonal antibody to USP1 were obtained from Merck Millipore (Darmstadt, Germany); mouse anti-human monoclonal antibody to SIK2 were obtained from BioLegend (San Diego, CA, USA); TRIzol reagent, Lipofectamine $^{\mathrm{TM}}$ 2000, Opti-MEM and the SuperScript III reverse transcriptase (RT) kit were obtained from Invitrogen Corp. (Carlsbad, CA, USA); Taq DNA polymerase was purchased from Fermentas, Inc. (Waltham, MA, USA); cell culture media and fetal bovine serum (FBS) were purchased from Gibco-BRL (Grand Island, NY, USA).

Tissue samples, cell lines and cell culture conditions. Tissue samples from 30 osteosarcomas were obtained from patients who underwent surgery at the Third Affiliated Hospital of Soochow University. All participants who had undergone surgical resection signed an informed consent form for this study. All osteosarcoma cases had been confirmed both clinically and pathologically. The experimental protocols for the present study were approved by the Hospital's Protection of Human Subjects Committee.

The human osteosarcoma U2OS cells were purchased from the Cell Bank of Chinese Academy of Sciences (Shanghai, China) in 2014 and were identified by short tandem repeat (STR) method in 2015. The cells were cultured in Dulbecco's modified Eagle's medium (DMEM) supplemented with $10 \%$ fetal bovine serum (FBS) and maintained at $37^{\circ} \mathrm{C}$ in a humidified atmosphere containing $5 \% \mathrm{CO}_{2}$.

Immunohistochemistry. Surgical specimens were fixed in $10 \%$ formalin solution and embedded in paraffin. Four- $\mu \mathrm{m}$ thick sections were deparaffinized, rehydrated and immersed in 3\% hydrogen peroxide solution for $15 \mathrm{~min}$ to block endogenous peroxidase activity. For antigen retrieval, the sections were boiled in EDTA buffer (pH 9.0) for $10 \mathrm{~min}$. Histologic sections were pre-treated in $10 \%$ normal goat serum in TBS for $30 \mathrm{~min}$ at room temperature in order to block non-specific binding sites. Then sections were incubated with polyclonal antibody against USP1 at a dilution of 1:100 in phosphate-buffered saline (PBS) at $4^{\circ} \mathrm{C}$ for $1 \mathrm{~h}$. After being rinsed five times with $\mathrm{PBS}$, sections were incubated with goat anti-mouse IgG conjugated with horseradish peroxidase at room temperature for $1 \mathrm{~h}$. Sections were developed with diaminobenzidene (DAB) as a chromogen and counterstained with haematoxylin. The sections were analyzed under light microscopy.

USP1 gene silence mediated by lentivirus-delivered shRNA. The human USP1-specific small interfering RNA (siRNA) sequence is 5'-GAAAGCTCCACATCAATAA-3', designed with an online Invitrogen software using the USP1 sequence (GenBank code:NM_003600) as a reference. The non-silencing sequence (5'-TTCTCCGAACGTGTCACGT-3') was used as a scrambled control. The recombinant lentiviral particles were regenerated in 293T cells by co-transfection of the modified pGCSIL-GFP viral vector together with the pHelper 1.0 and pHelper 2.0 plasmids using the Lipofectamine ${ }^{\mathrm{TM}} 2000$ reagent. The lentiviral particles were referred to as LV-USP1 siRNA or USP1-siRNA. We generated lentiviruses that express nonsilencing-shRNA as controls and named them scramble siRNA or scr-siRNA. The osteosarcoma cells transfected with the USP1-siRNA or scrambled siRNA were designated USP1siRNA and scr-siRNA, respectively. For lentiviral transduction, U2OS cells were cultured in 6-well plates at 70\% confluence and infected with control lentivirus or USP1-specific siRNA lentivirus at MOI of 20. After 5 days of infection, U2OS cells expressing GFP protein were observed by fluorescence microscopy to determine the infection efficiency.

SIK2 siRNA preparation. The siRNA sequences were designed by commercial software (Applied Biosystems/Ambion, Austin, TX, USA). For SIK2, the siRNA sense sequence is 5-CUA CCGAGAAGUACAAAUADTDT- 3 ' and the antisense sequence is 5'-UAUUUGUACUUCUCGGUAGdTdT-3'. The siRNA sequences employed as negative controls were $5^{\prime}$-GG CCUCAGCUGCGCGACGCdTdT-3' (sense) and 5'-GCGUC GCGCAGCUGGGCCAdTdT-3' (antisense). The selected sequences were confirmed by NCBI BLAST (http://www.ncbi. nlm.nih.gov/blast/) to make sure that the selected genes were specifically targeted. Chemically synthesized siRNAs were designed and sequenced by Guangzhou RiboBio, Co., Ltd. (Guangzhou, China).

SIK2 siRNA transfection. U2OS cells were transiently transfected with synthetic siRNA using Lipofectamine 2000 reagent according to the manufacturer's instructions. Briefly, U2OS cells were seeded onto 6-well plates in the maintenance medium at a density of $2 \times 10^{5}$ cells/well and allowed to grow for $12 \mathrm{~h}$. Then, $5 \mu \mathrm{l}$ Lipofectamine 2,000 and 200 pmol siRNA were diluted in serum-free medium to a total volume of $250 \mu \mathrm{l}$ and incubated for $20 \mathrm{~min}$ at room temperature. After the cells were washed with DMEM medium without FBS, the diluted 
siRNA-Lipofectamine mix was added to each well and incubated for $24 \mathrm{~h}$. Then the mix was replaced with fresh DMEM medium containing 10\% FBS. Forty-eight hours after the transfection, the U2OS cells were collected for protein and RNA isolation. The osteosarcoma cells transfected with the SIK2-siRNA or scrambled siRNA were designated SIK2siRNA and scr-siRNA, respectively.

Quantitative real-time PCR analysis. Total RNA was extracted from osteosarcoma cells using TRIzol reagent following the manufacturer's instructions. RNA samples (1 $\mu \mathrm{g} /$ reaction) were reverse-transcribed with the RevertAid First Strand cDNA Synthesis kit according to the manufacturer's recommended protocol. The RT reaction was used for amplification with Taq polymerase and a SYBR-Green RT-PCR kit (Takara, Kyoto, Japan) was used to detect specific real-time RT-PCR products. The resulting cDNA was amplified using specific primers, which were designed by using the GraphPad Prism 5.0 software (GraphPad Software, Inc., San Diego, CA, USA). The primer sequences were: For USP1, sense: 5'-AGGTTG CTAGTACAGCGTTTGC-3' and antisense: 5'-CACTGGATT CCTTGTTTCTATCAGA-3'; for SIK2, sense: 5'-AGTCTGA AGCCAGGGAAAA-3' and antisense: 5'-CATGTTGCCAGC AGTTCACC-3'; for $\beta$-actin, sense: 5'-GGCACTCTTCCAGC CTTCC-3' and antisense: 5'-GAGCCGCCGATCCACAC-3'. Relative gene-expression levels were calculated using the comparative $\mathrm{Ct}$ method, also called the $2^{-\Delta \Delta \mathrm{CT}}$ analysis method.

Cell proliferation assay. U2OS cells were seeded onto 96-well plates and incubated in corresponding media supplemented with $10 \%$ FBS. After 24, 48, 72 and $96 \mathrm{~h}$ of incubation, $10 \mu 1$ of Cell Counting kit-8 (CCK-8; Dojindo Laboratories, Kumamoto, Japan) was added into each well, followed by $4 \mathrm{~h}$ of incubation. The optical density values were measured at $450 \mathrm{~nm}$ using a Bio-Rad microplate reader to determine cell viability. The results were based on three independent experiments and are presented as mean \pm SD.

Colony-forming assay. Soft agar colony-formation assay was used to determine the effect of USP1 knockdown on the transformation capability of U2OS cells. In brief, U2OS cells were counted and inoculated in 6-well plates at a density of 3,000 cells/well. After being cultured at $37^{\circ} \mathrm{C}$ in $5 \% \mathrm{CO}_{2}$ in a humidified incubator for 2 weeks, U2OS cells were immobilized by $4 \%$ paraformaldehyde and stained using Giemsa dye for $20 \mathrm{~min}$. The cells were rinsed with distilled water. Plates were then scanned and photographed under an inverted microscope. All experiments were performed three times.

Flow cytometric analysis. The U2OS cells were harvested and washed with ice-cold PBS twice. Cells were then resuspended with $100 \mu \mathrm{l}$ of Annexin V binding buffer, and incubated with APC-labeled Annexin V at room temperature for $15 \mathrm{~min}$. Fluorescence-activated cell sorting (FACS) analysis for Annexin V staining was performed to determine cell apoptosis. Each experiment was performed in triplicate.

In vitro invasion assay. Twenty-four hours after the infection, the invasion ability of U2OS cells was determined using a Transwell chamber. A total of $40 \mu \mathrm{l}$ Matrigel $(10 \mathrm{mg} / \mathrm{ml})$
(BD Biosciences, San Jose, CA, USA) was coated to 48-well plates and polymerized at $37^{\circ} \mathrm{C}$ for $30 \mathrm{~min}$. U2OS cells were collected into DMEM medium, supplemented with $1 \%$ FBS. In brief, a $100 \mu \mathrm{l}$ cell suspension containing $2.0 \times 10^{5}$ cells was added to the upper chamber of each insert. For each Transwell chamber, $500 \mu 1$ of DMEM supplemented with $20 \%$ FBS was added in the lower chamber. After $24 \mathrm{~h}$ of incubation at $37^{\circ} \mathrm{C}$, cells and Matrigel on the upper surface of the well were removed. Cells invaded to the lower chamber were fixed with methanol, stained with $0.5 \%$ crystal violet for $20 \mathrm{~min}$ and then were counted under the light microscope. In addition, invaded cells were segregated, lysed and quantified at $570 \mathrm{~nm}$ using spectrophotometer to evaluate the amount of cells.

Western blot analysis. The U2OS cell pellets were lysed with protein extraction solution and incubated at $20^{\circ} \mathrm{C}$ for $20 \mathrm{~min}$. After protein quantification, the samples were separated by electrophoresis in SDS-PAGE and then transferred to a PVDF (polyvinylidene fluoride) membrane. After blocking in Trisbuffered saline Tween-20 (TBST) containing 5\% non-fat milk for $1 \mathrm{~h}$ at room temperature, membranes were subsequently incubated with primary antibodies against USP1, SIK2, matrix metalloproteinase-2 (MMP-2), glycogen synthase kinase-3 $\beta$ (GSK-3 $\beta$ ), B-cell lymphoma 2 (Bcl-2), signal transducer and activator of transcription 3 (Stat3), cyclin E1, Notch homolog 1 (Notch1), Wingless-type protein-1 (Wnt-1) and cyclin A1 at $4^{\circ} \mathrm{C}$ overnight. After incubation with secondary antibodies $(1: 1,000$; peroxidase-conjugated anti-mouse $\mathrm{IgG})$ for another $2 \mathrm{~h}$, the membranes were washed in TBST buffer and protein were detected using enhanced chemiluminescence. GAPDH and $\beta$-actin bands were used as loading controls.

Effect of MG132 on SIK2 protein levels. The U2OS cells were cultured in 6-well plates at a density of $4 \times 10^{4}$ cells/well. After $24 \mathrm{~h}$ of incubation, cells were treated with various concentrations of MG132 (2, 5 and $10 \mu \mathrm{mol} / \mathrm{l})$ and cultured for $0,4,8$ and $16 \mathrm{~h}$. Level of SIK 2 protein was determined using western blot analysis.

Statistical analysis. Data are reported as the mean \pm SEM of three independent experiments. Statistical analysis between comparable groups was performed using a one-way ANOVA by using GraphPad Prism 5.0 software. In each case, $\mathrm{P}<0.05$ was considered statistically significant.

\section{Results}

USP1 is overexpressed in osteosarcoma samples. We examined the expression of USP1 in 30 samples from patients with osteosarcoma. Of the 30 patients, $26(86.67 \%)$ were classified as positive expression of USP1. USP1 protein with positive staining was shown in the nuclei and cytoplasm of osteosarcoma tissues (Fig. 1A-a), while rare visible USP1 staining was detected in cartilage tumor tissues (Fig. 1A-b) and normal bone tissues (Fig. 1A-c). The USP1 expression in osteosarcoma tissues was significantly higher than that in cartilage tumor tissues and normal bone tissues.

Construction of a lentiviral vector mediating RNAi targeting of USP1 (LV-USPI siRNA) and its effects on USP1 expres- 
A

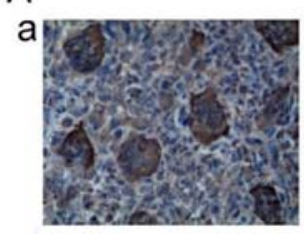

B

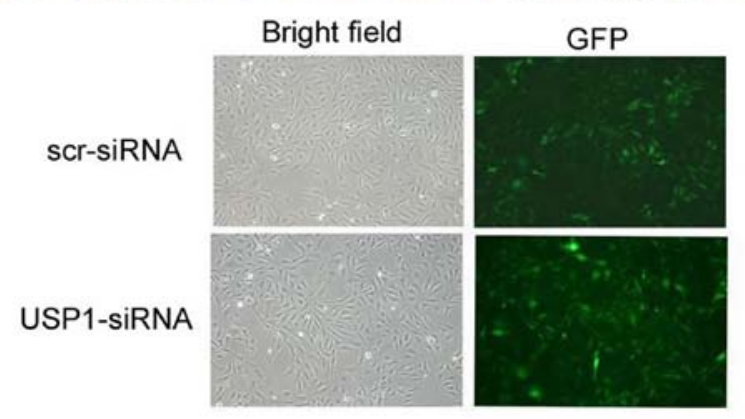

C

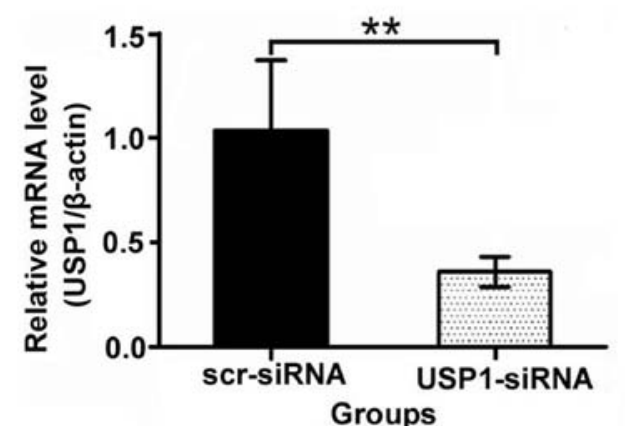

D

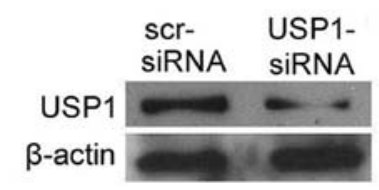

Figure 1. Analysis for expression of USP1 in osteosarcoma tissues and lentivirus infection in U2OS cells. (A) Immunohistochemistry analysis. Immunohistochemistry analysis of USP1 protein expression in osteosarcoma tissues (a), cartilage tumor tissues (b) and normal bone tissues (c) (magnification, x400). (B) Lentivirus infection in osteosarcoma cells. Green fluorescence indicates the successful delivery of siRNA into U2OS cells (magnification, $\mathrm{x} 400$ ). (C) The USP1 mRNA expression level was quantified by real-time RT-PCR. USP1 siRNA significantly decreased the level of USP1 mRNA in U2OS cells. (D) The USP1 protein expression level was quantified by western blot analysis. USP1 siRNA significantly decreased the level of USP1 protein in U2OS cells. ${ }^{*} \mathrm{P}<0.05,{ }^{* *} \mathrm{P}<0.01$, in comparison to the scrsiRNA group.

sion. To further illuminate the relationship between USP1 and osteosarcoma, we constructed a lentivirus-based USP1specific siRNA vector and a scramble-siRNA vector. The two vectors were infected into U2OS cells for 3 days and $>90 \%$ of the cells showed a green fluorescence indicating successful infection (Fig. 1B). To confirm whether the USP1-siRNA had silenced the expression of USP1 mRNA and protein, real-time PCR and western blot analysis were performed on lentivirus-infected cells. The results showed that both mRNA (Fig. 1C) and protein (Fig. 1D) expression levels of USP1 were significantly reduced by infection of USP1-siRNA, compared to scr-siRNA. These data suggest that the infection with USP1-siRNA effectively downregulates USP1 expression in U2OS cells.
Effects of USPI-siRNA on cell viability. CCK-8 assay was performed to study the effect of USP1-siRNA on U2OS cell growth. As shown in Fig. 2A, the cell growth of USP1-siRNA groups showed a significant $(\mathrm{P}<0.01)$ reduction in cell viability in comparison to the scr-siRNA groups. The result of a colony formation assay showed that the number of colonies of the USP1-siRNA group $(2249.33 \pm 156.31)$ was significantly less than that of the scr-siRNA group (2751.00 \pm 83.07$)$ in U2OS cells $(\mathrm{P}<0.01)$ (Fig. 2B). These results demonstrate that the reduction in USP1 expression decreased the ability of U2OS cells to form colonies. Furthermore, these results suggest that cell proliferation is reduced due to the silencing of the USP1 gene.

USP1 silencing induces apoptosis in U2OS cells. To determine whether USP1 depletion induced cell apoptosis, flow cytometry was used after the silencing of USP1. The flow cytometric analysis shows that the percentage of apoptotic $\mathrm{U} 2 \mathrm{OS}$ cells was $3.88 \pm 0.22 \%$ in the scr-siRNA group cells, and the percentage of apoptotic cells increased to $20.57 \pm 0.64 \%$ in the USP1-siRNA group cells $(\mathrm{P}<0.01)$ (Fig. 2C). These data suggest that the depletion of USP1 specifically induces apoptosis of the U2OS cells.

Depletion of USP1 inhibits the invasion of U2OS cells. Next, we performed an in vitro invasion assay, the results showed that OD value of $570 \mathrm{~nm}$ was $0.27 \pm 0.04$ in the scr-siRNA group and $0.17 \pm 0.01$ in the USP1-siRNA group $(\mathrm{P}<0.01)$ (Fig. 2D). These results demonstrate that USP1-siRNA could reduce the invasion of U2OS cells.

Western blot analysis. The protein levels of related molecules were also examined. The results showed that USP1 knockdown downregulated SIK2, MMP-2, GSK-3 $\beta$, Bcl-2, cyclin E1, Notch1, Stat3, cyclin A1 and Wnt-1 in U2OS cells (Fig. 3A). These results suggest that USP1 downregulation inhibits growth and invasion of $\mathrm{U} 2 \mathrm{OS}$ cells through these proteins.

MG132 affects SIK2 protein level. We further examined whether proteasome inhibitor MG132 can increase the level of SIK2 protein in U2OS cells. The result showed that cells treated with MG132 $(5 \mu \mathrm{mol} / \mathrm{l})$ for 4 or $8 \mathrm{~h}$ exhibited an elevation of SIK2 protein (Fig. 3B). It suggests that MG132 upregulates SIK2 expression by decreasing its protein degradation, and therefore, inhibition of proteasome function increases SIK2 protein level in U2OS cells.

SIK2 siRNA significantly inhibits the $m R N A$ and protein expression of SIK2. Real-time PCR and western blot analysis were performed to confirm whether the SIK2 siRNA inhibited the expression of SIK 2 mRNA and protein. The results showed that SIK2 mRNA and protein expression in SIK2-siRNA group were significantly inhibited compared to scr-siRNA group as shown in Fig. 4A and B. It indicates that the targeted SIK2 siRNA inhibited significantly the expression of SIK2 gene.

Downregulation of SIK2 suppresses colony formation in U2OS cells. Colony-forming assays were performed to determine the effect of SIK2 deletion on colony formation. The 

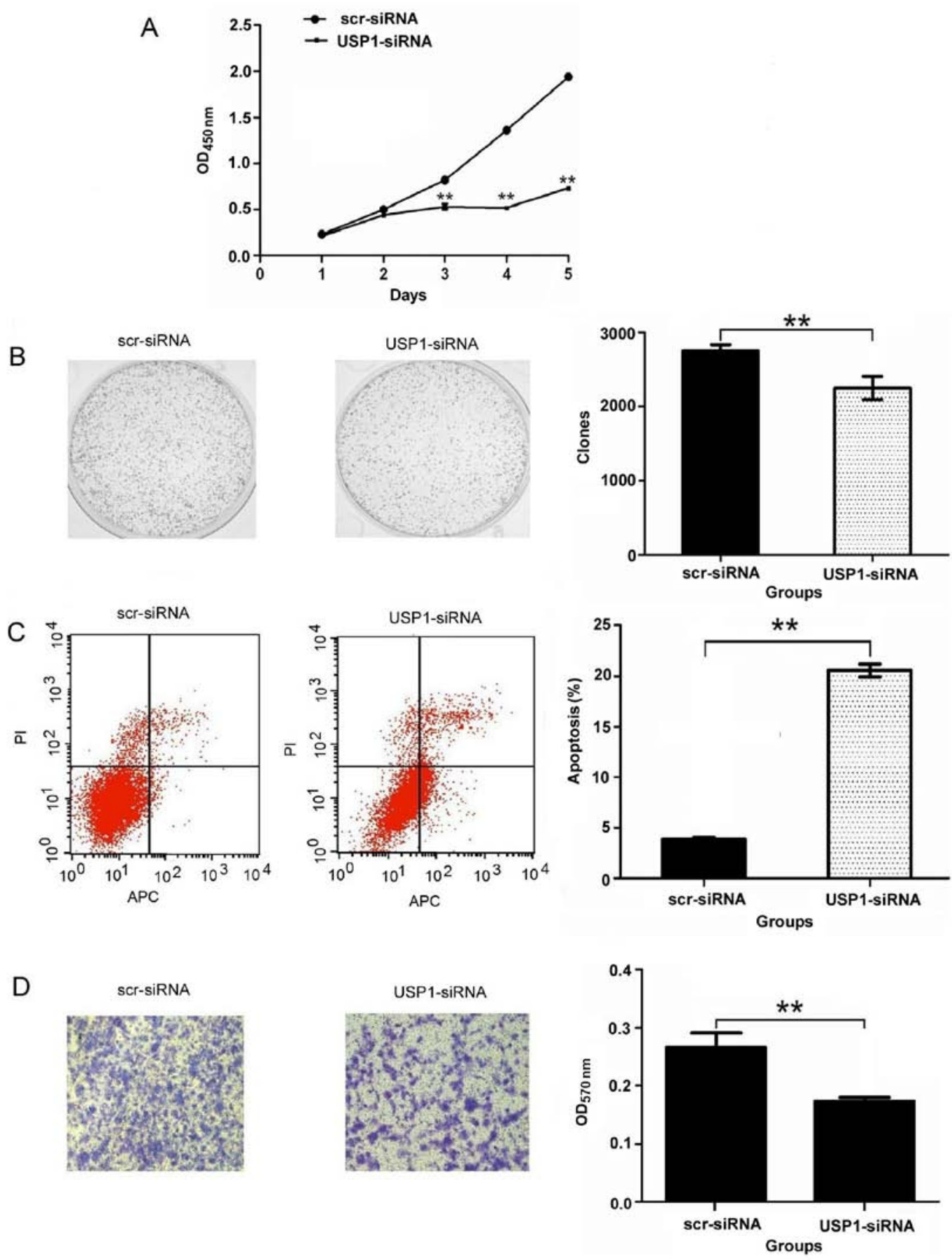

Figure 2. Silencing effect of USP1 siRNA in U2OS cells. (A) Effect of USP1 silencing on the proliferation of osteosarcoma cells. The impact of USP1 suppression on proliferation of U2OS cells was assessed by CCK-8 assay, USP1 siRNA depressed the growth curve of U2OS cells as compared with the scr-siRNA group. (B) Inhibition of USP1 decreased colony formation of U2OS cells. Colony formation assay showed that USP1 siRNA decreased the clone numbers of U2OS cells as compared with scr-siRNA group. (C) The effects of USP1 siRNA on apoptosis of U2OS cells was determined by flow cytometric analysis. Flow cytometric analysis showed that knockdown of USP1 expression increased cell apoptosis in U2OS cells. (D) Effects of USP1 siRNA on invasion of U2OS cells. Depletion of USP1 inhibits the invasion of U2OS cells. ${ }^{*} \mathrm{P}<0.05,{ }^{* *} \mathrm{P}<0.01$ in comparison to the scr-siRNA group.

results showed that the number of colonies of the SIK2-siRNA group (1492.00 \pm 74.65$)$ was significantly less than that of the scr-siRNA group $(1713.00 \pm 159.46)$ in $\mathrm{U} 2 \mathrm{OS}$ cells $(\mathrm{P}<0.01)$ (Fig. 4C). This clearly demonstrates that the number of the colonies decreased significantly with the SIK2 deletion.

SIK2 silencing induces apoptosis in U2OS cells. The flow cytometric analysis shows that the percentage of apoptotic U2OS cells was $8.05 \pm 0.20 \%$ in the scr-siRNA group cells, and the percentage of apoptotic cells increased to $27.09 \pm 3.07 \%$ in the SIK2-siRNA group cells $(\mathrm{P}<0.01)$ (Fig. 4D). It indicates that the percentage of apoptotic cells increased significantly with the SIK2 deletion.

SIK2 is involved in U2OS cell invasion. The images of cells stained with crystal violet suggests that knocking down the expression of SIK2 resulted in the decrease of the amount of invaded cells. Additional observation of absorption assay also 


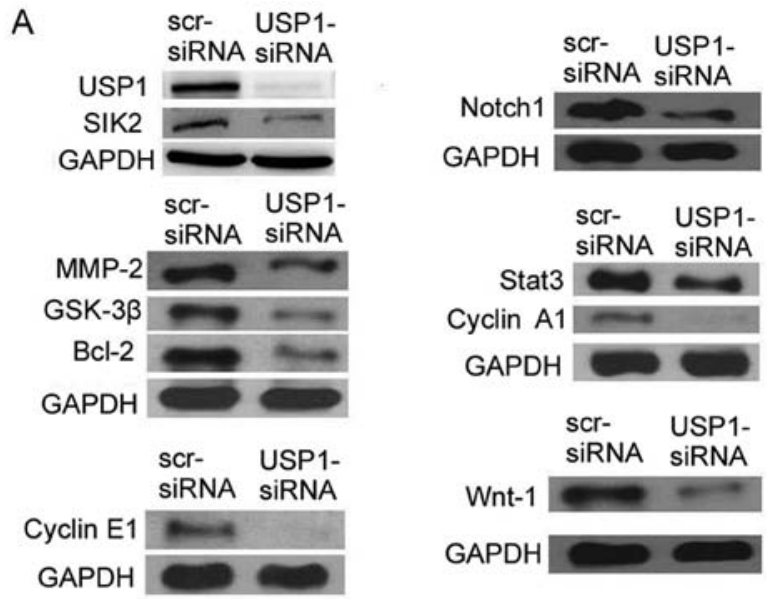

B

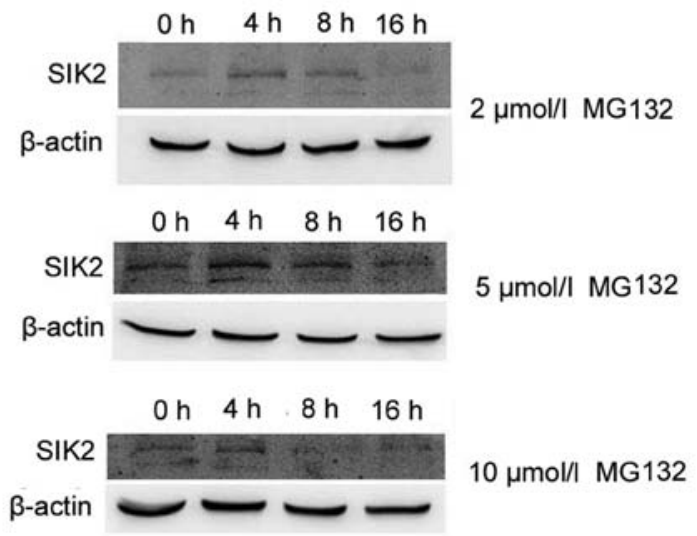

Figure 3. Western blot analysis. (A) The protein levels of related molecules Protein expression levels of USP1, SIK2, MMP-2, GSK-3 $\beta$, Bcl-2, cyclin E1, Notch1, Stat3, cyclin A1 and Wnt-1 were significantly decreased after USP1 silencing. (B) MG132 affects SIK2 protein level in U2OS cells. U2OS cells treated with MG132 $(5 \mu \mathrm{mol} / \mathrm{l})$ for 4 or $8 \mathrm{~h}$ exhibited an elevation of SIK2 protein.

proved that the migration ability of U2OS cells reduced after knocking down the expression of SIK2. The results showed that OD value of $570 \mathrm{~nm}$ was $0.13 \pm 0.02$ in the SIK2-siRNA group and is significantly less than the reading in the scrsiRNA group $(0.19 \pm 0.01, \mathrm{P}<0.01)$ (Fig. $4 \mathrm{E})$, all of these data suggest that SIK2 was involved in the invasion of U2OS cells.

\section{Discussion}

Osteosarcoma occurs most frequently at the metaphysis of long bones during longitudinal growth spurts in children and young adults (19). The treatment and prognosis for this disease still need to be improved.

USP1 belongs to the cysteine protease family, the USP1 gene encodes a 785 amino acid protein with a predicted molecular weight of $88.2 \mathrm{kDa}(20)$. USP1 contains the conserved USP domain that characterizes this DUB family, with an aminoterminal Cys box motif and a carboxy-terminal, His box motif that contain the catalytic residues (Cys90, His593 and Asp751) $(15,21)$. Notably, it has been uncovered that the catalytic activity of USP1 is stimulated through the formation of a tight complex with a WD40 repeat protein UAF1 (22). UAF1 plays a critical role as a cofactor and UAF1 binding induces conformational changes in USP1 active site increasing the enzyme activity dramatically by stabilizing it. Importantly, studies show that several inhibitors of the USP1/UAF1 complex act synergistically with cisplatin in cancer-derived cell lines, indicating that this complex may represent a potential therapeutic target in cancer (23-25).

DUBs play crucial roles in cancer development, progression and metastasis (26). Targeting DUBs is a new strategy for anti-tumorigenic therapeutics (27). USP1-null mice were hypersensitive to DNA damage, it is likely that targeting USP1 could increase the sensitivity of cancer cells to DNA damaging agents (28).

In the present study, we found that silencing of USP1 could inhibit osteosarcoma cell proliferation and invasion, which provides direct evidence that USP1 may serve as a target for such tumor treatment. The detailed mechanisms underlying how USP1 functions in osteosarcoma still need to be elucidated in the future.

Salt-inducible kinase 2 (SIK2) is a multifunctional serine/ threonine kinase of the AMP-activated protein kinase (AMPK) sub-family, and it plays a role in cAMP response elementbinding protein (CREB)-mediated gene transcription. Previous studies have demonstrated that increased SIK2 expression is significantly correlated with poor prognosis in overall survival in patients with high-grade serous ovarian cancer (HGSC). Recent reports have shown that SIK2 is required for bipolar mitotic spindle formation and is a potential target for ovarian cancer therapy. Examination of SIK2 found that it regulates mitotic progression and transcription in prostate cancer cells. Knockdown of SIK2 expression inhibits growth and induces cell cycle arrest and apoptosis $(29,30)$. The results showed that knockdown of USP1 could downregulate SIK2.

MG132 is a specific proteasome inhibitor and reduces the degradation of ubiquitin-conjugated proteins in mammalian cells (31). In order to explore the relationship between USP1 and SIK2, the U2OS cells were treated with MG132. The study found that MG132 could upregulate the expression of SIK2, it suggests that USP1 may stabilize the expression of SIK2 through protein ubiquitination. In the present study, we also found that knockdown of SIK 2 could inhibit the ability of forming colonies, induce apoptosis and reduce the invasiveness of osteosarcoma cells, which indicates the essential roles of SIK2 in such tumors.

MMP-2, an important member of the MMP family, is able to degrade extracellular matrix components to promote cancer cell migration and invasion in multiple tumor types (32-34). GSK-3 $\beta$ is a ubiquitous serine/threonine kinase that plays different roles in different types of cancers (35). The GSK-3 $\beta$ gene may function as an oncogene in various types of human cancer, including colon cancer and osteosarcoma $(36,37)$. Bcl-2, a kind of classic proto-oncogene, plays an important regulatory role in many kinds of malignant tumor cells apoptosis and distant metastasis, such as liver, lung, kidney, bladder and gastric cancer (38). Experimental and pathological evidence shows that cyclin E1 deregulation is oncogenic $(39,40)$. Elevated cyclin E1 is associated with aggressive disease in a variety of human tumors (41). The Notch signaling pathway is shown to be an important driver of tumor growth and metastasis and dysregulated in many cancers $(42,43)$. Notch 1 is required for multiple 
A
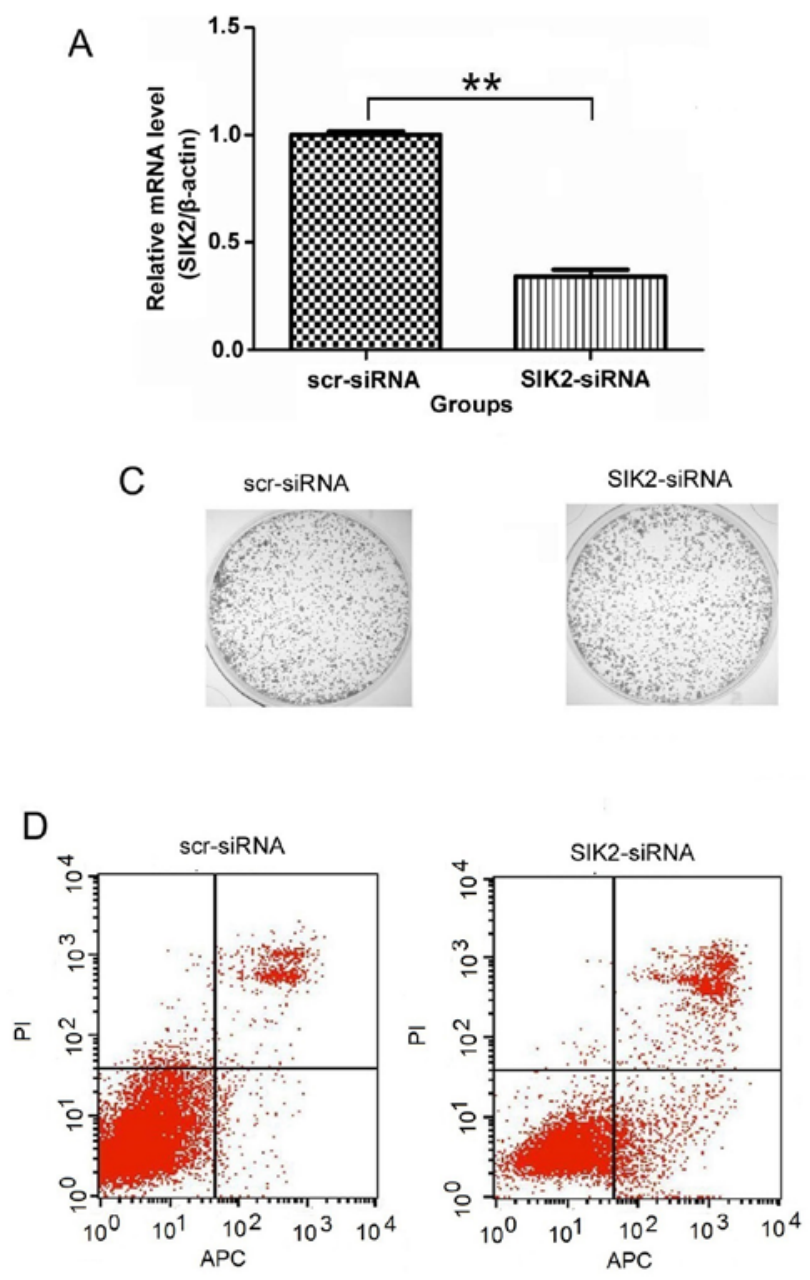

E

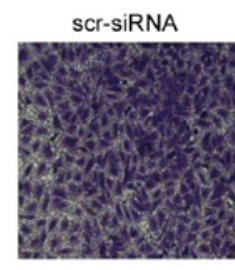

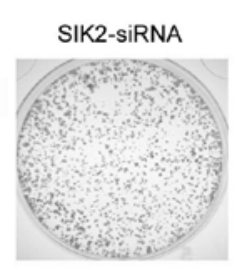

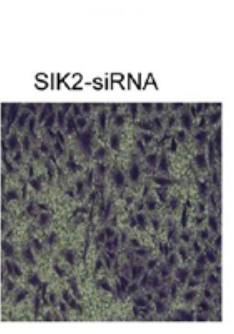

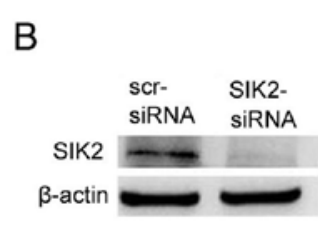
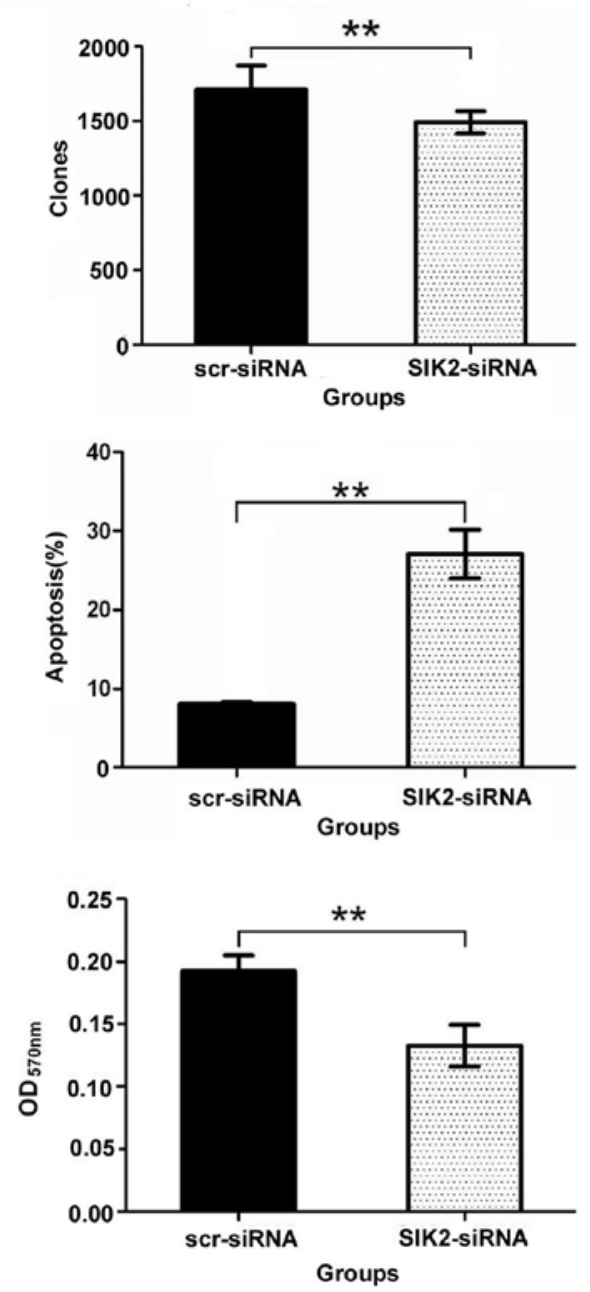

Figure 4. Silencing effect of SIK2 siRNA in U2OS cells. (A) The SIK2 mRNA expression level was quantified by real-time RT-PCR. SIK2 siRNA significantly decreased the level of SIK2 mRNA in U2OS cells. (B) The SIK2 protein expression level was quantified by western blot analysis. SIK2 siRNA significantly decreased the level of SIK2 protein in U2OS cells. (C) Inhibition of SIK2 decreased colony formation of U2OS cells. Colony formation assay showed that SIK2 siRNA decreased the clone numbers of U2OS cells as compared with scr-siRNA group. (D) The effects of SIK2 siRNA on apoptosis of U2OS cells was determined by flow cytometric analysis. Flow cytometric analysis showed that knockdown of SIK2 expression increased cell apoptosis in U2OS cells. (E) Effects of SIK2 siRNA on invasion of U2OS cells. Depletion of SIK2 inhibits the invasion of U2OS cells. ${ }^{*} \mathrm{P}<0.05,{ }^{* *} \mathrm{P}<0.01$ in comparison to the scr-siRNA group.

cell fate determination processes, the deregulation of the Notch1 signaling cascade plays a crucial role in some solid tumors (44). Stat3, a central cytoplasmic transcription factor, is an important regulator of many biological processes (45). It regulates a number of genes that are critical to tumor cell proliferation, invasion, metastasis, angiogenesis and immune evasion $(46,47)$. Previous studies revealed that cyclin A1 is highly expressed in many types of cancer, such as cancers of breast, lung and prostate (48-50). As a member of Wingless family and a secreted glycoprotein, Wnt-1 binds and activates the frizzled receptor, triggering a signaling cascade through
GSK-3 $\beta$ and adenomatous polyposis coli protein (APC). Some cancer cells show upregulation of Wnt-1 signaling and continue to metastasize (51-53).

Notably, in the present study, all the above factors were reduced after knowdown of USP1 expression, which indicated that the inhibition of proliferation and invasion of human osteosarcoma cells after knockdown of USP1 expression is through reducing expression of these factors. Further experiments are required.

It is important to note that USP1 expression is increased in osteosarcoma tissues compared to cartilage tumor tissues and 
normal bone tissues. This suggests an association between the overexpression of USP1 and osteosarcoma genesis.

In summary, our results suggest that silencing of USP1 inhibits cell proliferation and invasion in osteosarcoma cells in vitro, and reduce some tumor-related gene expression, which in turn provides the potential mechanisms of how UPS1 functions in osteosarcoma cells, and therapeutic targets for such tumors in the future.

\section{Acknowledgements}

The present study was supported by grants from the Natural Science Foundation of Jiangsu Province (BK20151177), the National Science Foundation of China (81471263), the Suzhou Planning Project of Science and Technology (SYS201301), the Natural Science Foundation for the Youth (no. 81402220) and the Youth Scientific Research Project of Wuxi Health Bureau (Q201412). The authors would like to thank Dr Wenxiang Wei (Soochow University, Suzhou 215123, China) for his sincere help and technical support.

\section{References}

1. Endo-Munoz L, Evdokiou A and Saunders NA: The role of osteoclasts and tumour-associated macrophages in osteosarcoma metastasis. Biochim Biophys Acta 1826: 434-442, 2012.

2. Poletajew S, Fus L and Wasiutyński A: Current concepts on pathogenesis and biology of metastatic osteosarcoma tumors. Ortop Traumatol Rehabil 13: 537-545, 2011.

3. Allison DC, Carney SC, Ahlmann ER, Hendifar A, Chawla S, Fedenko A, Angeles C and Menendez LR: A meta-analysis of osteosarcoma outcomes in the modern medical era. Sarcoma 2012: 704872, 2012.

4. Yao C, Wei JJ, Wang ZY, Ding HM, Li D, Yan SC, Yang YJ and Gu ZP: Perifosine induces cell apoptosis in human osteosarcoma cells: New implication for osteosarcoma therapy? Cell Biochem Biophys 65: 217-227, 2013.

5. Chen ZJ and Sun LJ: Nonproteolytic functions of ubiquitin in cell signaling. Mol Cell 33: 275-286, 2009.

6 . Komander D: The emerging complexity of protein ubiquitination. Biochem Soc Trans 37: 937-953, 2009.

7. Fraile JM, Quesada V, Rodríguez D, Freije JM and López-Otín C: Deubiquitinases in cancer: New functions and therapeutic options. Oncogene 31: 2373-2388, 2012.

8. García-Santisteban I, Peters GJ, Giovannetti E and Rodríguez JA: USP1 deubiquitinase: Cellular functions, regulatory mechanisms and emerging potential as target in cancer therapy. Mol Cancer 12: 91, 2013

9. Williams SA, Maecker HL, French DM, Liu J, Gregg A, Silverstein LB, Cao TC, Carano RA and Dixit VM: USP1 deubiquitinates ID proteins to preserve a mesenchymal stem cell program in osteosarcoma. Cell 146: 918-930, 2011.

10. Liu Y, Luo X, Hu H, Wang R, Sun Y, Zeng R and Chen H: Integrative proteomics and tissue microarray profiling indicate the association between overexpressed serum proteins and non-small cell lung cancer. PLoS One 7: e51748, 2012.

11. Kim H and D'Andrea AD: Regulation of DNA cross-link repair by the Fanconi anemia/BRCA pathway. Genes Dev 26: 1393-1408, 2012.

12. Huang TT, Nijman SM, Mirchandani KD, Galardy PJ, Cohn MA, Haas W, Gygi SP, Ploegh HL, Bernards R and D'Andrea AD: Regulation of monoubiquitinated PCNA by DUB autocleavage. Nat Cell Biol 8: 339-347, 2006.

13. Fox JT, Lee KY and Myung K: Dynamic regulation of PCNA ubiquitylation/deubiquitylation. FEBS Lett 585: 2780-2785, 2011.

14. Cohn MA, Kowal P, Yang K, Haas W, Huang TT, Gygi SP and D'Andrea AD: A UAF1-containing multisubunit protein complex regulates the Fanconi anemia pathway. Mol Cell 28: 786-797, 2007.

15. Villamil MA, Chen J, Liang Q and Zhuang Z: A noncanonical cysteine protease USP1 is activated through active site modulation by USP1-associated factor 1. Biochemistry 51: 2829-2839, 2012.
16. Yang K, Moldovan GL, Vinciguerra P, Murai J, Takeda S and D'Andrea AD: Regulation of the Fanconi anemia pathway by a SUMO-like delivery network. Genes Dev 25: 1847-1858, 2011.

17. Davidson BL and McCray PB Jr: Current prospects for RNA interference-based therapies. Nat Rev Genet 12: 329-340, 2011.

18. Angaji SA, Hedayati SS, Poor RH, Madani S, Poor SS and Panahi S: Application of RNA interference in treating human diseases. J Genet 89: 527-537, 2010.

19. Mirabello L, Troisi RJ and Savage SA: Osteosarcoma incidence and survival rates from 1973 to 2004: data from the Surveillance, Epidemiology, and End Results Program. Cancer 115: 1531-1543, 2009.

20. Fujiwara T, Saito A, Suzuki M, Shinomiya H, Suzuki T, Takahashi E, Tanigami A, Ichiyama A, Chung CH, Nakamura Y, et al: Identification and chromosomal assignment of USP1, a novel gene encoding a human ubiquitin-specific protease. Genomics 54: 155-158, 1998.

21. Békés M, Okamoto K, Crist SB, Jones MJ, Chapman JR, Brasher BB, Melandri FD, Ueberheide BM, Denchi EL and Huang TT: DUB-resistant ubiquitin to survey ubiquitination switches in mammalian cells. Cell Rep 5: 826-838, 2013.

22. Cohn MA, Kee Y, Haas W, Gygi SP and D'Andrea AD: UAF1 is a subunit of multiple deubiquitinating enzyme complexes. J Biol Chem 284: 5343-5351, 2009.

23. Chen J, Dexheimer TS, Ai Y, Liang Q, Villamil MA, Inglese J, Maloney DJ, Jadhav A, Simeonov A and Zhuang Z: Selective and cell-active inhibitors of the USP1/ UAF1 deubiquitinase complex reverse cisplatin resistance in non-small cell lung cancer cells. Chem Biol 18: 1390-1400, 2011.

24. Liang Q, Dexheimer TS, Zhang P, Rosenthal AS, Villamil MA, You C, Zhang Q, Chen J, Ott CA, Sun H, et al: A selective USP1-UAF1 inhibitor links deubiquitination to DNA damage responses. Nat Chem Biol 10: 298-304, 2014.

25. Wahi D, Jamal S, Goyal S, Singh A, Jain R, Rana P and Grover A: Cheminformatics models based on machine learning approaches for design of USP1/UAF1 abrogators as anticancer agents. Syst Synth Biol 9: 33-43, 2015.

26. McClurg UL and Robson CN: Deubiquitinating enzymes as oncotargets. Oncotarget 6: 9657-9668, 2015.

27. D'Arcy P and Linder S: Molecular pathways: Translational potential of deubiquitinases as drug targets. Clin Cancer Res 20: 3908-3914, 2014.

28. Park E, Kim JM, Primack B, Weinstock DM, Moreau LA, Parmar K and D'Andrea AD: Inactivation of Uaf 1 causes defective homologous recombination and early embryonic lethality in mice. Mol Cell Biol 33: 4360-4370, 2013.

29. Ahmed AA, Lu Z, Jennings NB, Etemadmoghadam D, Capalbo L, Jacamo RO, Barbosa-Morais N, Le XF, Vivas-Mejia P, LopezBerestein G, et al; Australian Ovarian Cancer Study Group: SIK2 is a centrosome kinase required for bipolar mitotic spindle formation that provides a potential target for therapy in ovarian cancer. Cancer Cell 18: 109-121, 2010.

30. Bon H, Wadhwa K, Schreiner A, Osborne M, Carroll T, RamosMontoya A, Ross-Adams H, Visser M, Hoffmann R, Ahmed AA, et al: Salt-inducible kinase 2 regulates mitotic progression and transcription in prostate cancer. Mol Cancer Res 13: 620-635, 2015.

31. Jamart C, Raymackers JM, Li An G, Deldicque L and Francaux M: Prevention of muscle disuse atrophy by MG132 proteasome inhibitor. Muscle Nerve 43: 708-716, 2011.

32. Aparna M, Rao L, Kunhikatta V and Radhakrishnan R: The role of MMP-2 and MMP-9 as prognostic markers in the early stages of tongue squamous cell carcinoma. J Oral Pathol Med 44: 345-352, 2015.

33. Yang W, Li Q and Pan Z: Sphingosine-1-phosphate promotes extravillous trophoblast cell invasion by activating MEK/ERK/ MMP-2 signaling pathways via S1P/S1PR1 axis activation. PLoS One 9: e106725, 2014.

34. Chen SJ, Yao XD, Peng BO, Xu YF, Wang GC, Huang J, Liu M and Zheng JH: Epigallocatechin-3-gallate inhibits migration and invasion of human renal carcinoma cells by downregulating matrix metalloproteinase-2 and matrix metalloproteinase-9. Exp Ther Med 11: 1243-1248, 2016.

35. Kotliarova S, Pastorino S, Kovell LC, Kotliarov Y, Song H, Zhang W, Bailey R, Maric D, Zenklusen JC, Lee J, et al: Glycogen synthase kinase-3 inhibition induces glioma cell death through c-MYC, nuclear factor-kappaB, and glucose regulation. Cancer Res 68: 6643-6651, 2008. 
36. Salim T, Sjölander A and Sand-Dejmek J: Nuclear expression of glycogen synthase kinase- $3 \beta$ and lack of membranous $\beta$-catenin is correlated with poor survival in colon cancer. Int J Cancer 133 : $807-815,2013$.

37. Tang QL, Xie XB, Wang J, Chen Q, Han AJ, Zou CY, Yin JQ, Liu DW, Liang Y, Zhao ZQ, et al: Glycogen synthase kinase-3 $\beta$, $\mathrm{NF}-\mathrm{kB}$ signaling, and tumorigenesis of human osteosarcoma. J Natl Cancer Inst 104: 749-763, 2012.

38. Yao K, Xing HC, Wu B, Li Y, Liao AJ, Yang W and Liu ZG: Effect of TIEG1 on apoptosis and expression of Bcl-2/Bax and Pten in leukemic cell lines. Genet Mol Res 14: 1968-1974, 2015.

39. Hwang HC and Clurman BE: Cyclin E in normal and neoplastic cell cycles. Oncogene 24: 2776-2786, 2005.

40. Spruck CH, Won KA and Reed SI: Deregulated cyclin E induces chromosome instability. Nature 401: 297-300, 1999.

41. Hunt KK and Keyomarsi K: Cyclin E as a prognostic and predictive marker in breast cancer. Semin Cancer Biol 15: 319-326, 2005.

42. Arcaroli JJ, Quackenbush KS, Purkey A, Powell RW, Pitts TM, Bagby S, Tan AC, Cross B, McPhillips K, Song EK, et al: Tumours with elevated levels of the Notch and Wnt pathways exhibit efficacy to PF-03084014, a $\gamma$-secretase inhibitor, in a preclinical colorectal explant model. Br J Cancer 109: 667-675, 2013.

43. Takebe N, Miele L, Harris PJ, Jeong W, Bando H, Kahn M, Yang SX and Ivy SP: Targeting Notch, Hedgehog, and Wnt pathways in cancer stem cells: Clinical update. Nat Rev Clin Oncol 12: 445-464, 2015

44. Ranganathan P, Weaver KL and Capobianco AJ: Notch signalling in solid tumours: A little bit of everything but not all the time. Nat Rev Cancer 11: 338-351, 2011.
45. Darnell JE Jr, Kerr IM and Stark GR: Jak-STAT pathways and transcriptional activation in response to IFNs and other extracellular signaling proteins. Science 264: 1415-1421, 1994.

46. Yu H, Pardoll D and Jove R: STATs in cancer inflammation and immunity: A leading role for STAT3. Nat Rev Cancer 9: 798-809, 2009.

47. Hajimoradi M, Mohammad Hassan Z, Ebrahimi M, Soleimani M, Bakhshi M, Firouzi J and Samani FS: STAT3 is overactivated in gastric cancer stem-like cells. Cell J 17: 617-628, 2016.

48. Shames DS, Girard L, Gao B, Sato M, Lewis CM, Shivapurkar N, Jiang A, Perou CM, Kim YH, Pollack JR, et al: A genome-wide screen for promoter methylation in lung cancer identifies novel methylation markers for multiple malignancies. PLoS Med 3: e486, 2006

49. Syed Khaja AS, Dizeyi N, Kopparapu PK, Anagnostaki L, Härkönen P and Persson JL: Cyclin A1 modulates the expression of vascular endothelial growth factor and promotes hormonedependent growth and angiogenesis of breast cancer. PLoS One 8: e72210, 2013.

50. Wegiel B, Bjartell A, Ekberg J, Gadaleanu V, Brunhoff C and Persson JL: A role for cyclin A1 in mediating the autocrine expression of vascular endothelial growth factor in prostate cancer. Oncogene 24: 6385-6393, 2005.

51. Polakis P: Wnt signaling and cancer. Genes Dev 14: 1837-1851, 2000.

52. van Gijn ME, Daemen MJ, Smits JF and Blankesteijn WM: The wnt-frizzled cascade in cardiovascular disease. Cardiovasc Res 55: 16-24, 2002

53. Kim YM, Kim IH and Nam TJ: Capsosiphon fulvescens glycoprotein inhibits AGS gastric cancer cell proliferation by downregulating Wnt-1 signaling. Int J Oncol 43: 1395-1401, 2013. 